\title{
A matched case-control study to assess the carbapenem- resistant Enterobacteriaceae infections among hospitalized children at King Fahad Medical City, Riyadh, Saudi Arabia
}

Omar A. Alzomor, MD, Tariq S. Alfawaz, MD, Amani Abu-Shaheen, MPH,

Mohammed A. Alshehri, MD, Dayel Al Shahrani, MD, CPHQ.

\section{ABSTRACT}

$$
\begin{aligned}
& \text { الأهداف : لتحديد عو امل الخطر المرتبطة بالتهابات الأمعاء والبكتيريا }
\end{aligned}
$$

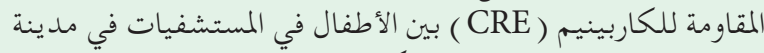

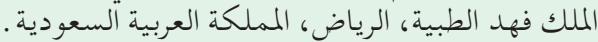

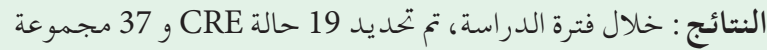

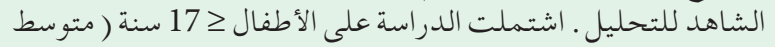

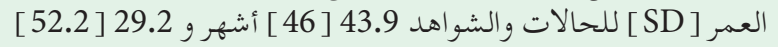

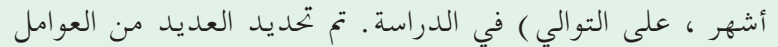

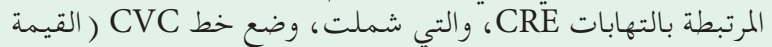

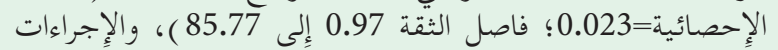

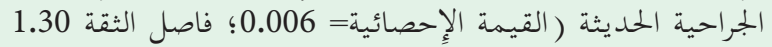

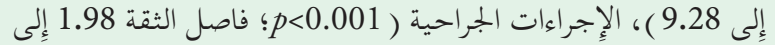

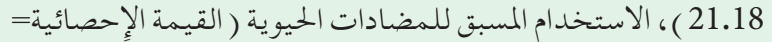

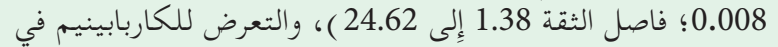
الأشهر الثلاثة الماضية (القيمة الإِحصائية=

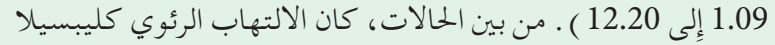

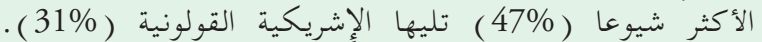

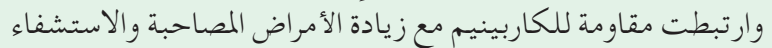

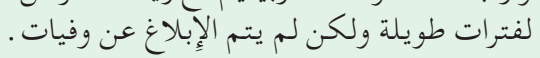

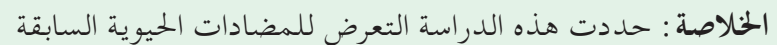

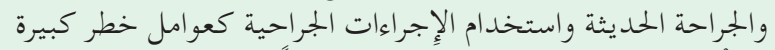

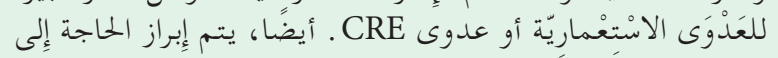

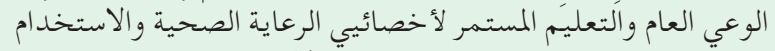

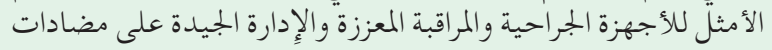

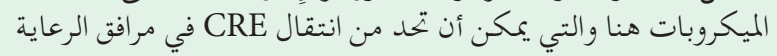
الصحية.

Objectives: To identify risk factors associated with carbapenem-resistant Enterobacteriaceae (CRE) infections among hospitalized children at King Fahad Medical City, Riyadh, Saudi Arabia.
Methods: A retrospective matched case-control study was conducted in pediatric patients with CRE infection at King Fahad Medical City, Riyadh, Saudi Arabia between January 2016-2017.

Results: During the study period, 19 CRE cases and 37 controls were identified for analysis. Children $\leq 17$ years (mean age \pm SD for cases was $43.9 \pm 46$ months and controls was $29.2 \pm 52.2$ months) were included in the study. Several factors associated with CRE infections were identified, which included, central venous catheter (CVC) line placement $(p=0.023$; confidence interval $[\mathrm{CI}]$ : $0.97-85.77)$, recent surgical procedures $(p=0.006 ; \quad$ CI: $1.30-9.28)$, invasive procedures $(p<0.001$; CI: $1.98-21.18)$, use of prior antibiotics $(p=0.008$; CI: $1.38-24.62)$, and carbapenem exposure in the past 3 months ( $p=0.004$; CI: 1.09-12.20). Among the cases, Klebsiella pneumonia was the most commonly identified (47\%) followed by Escherichia coli (31\%). Carbapenem-resistant Enterobacteriaceae was associated with increased comorbidities and prolonged hospitalization however, no mortalities were reported.

Conclusion: This study identified prior antibiotic exposure, recent surgery and the use of invasive procedures as significant risk factors for colonization or infection with CRE. Also, the need for public awareness, continuing education for healthcare professionals, optimum use of invasive devices, enhanced surveillance, and antimicrobial stewardship are highlighted here which can limit CRE transmission in healthcare facilities.

Saudi Med J 2019; Vol. 40 (11): 1105-1110 doi: 10.15537/smj.2019.11.24586

From the Pediatric Infectious Diseases Section (Alzomor, Alfawaz, Alshehri, Al Shahrani), Children's Specialized Hospital, and from the Department of Scientific Writing Research Services (Abu-Shaheen), Administration Research Center, King Fahad Medical City, Riyadh, Kingdom of Saudi Arabia.

\section{Received 18th March 2019. Accepted 17th September 2019.}

Address correspondence and reprint request to: Dr. Omar A. Alzomor Pediatric Infectious Diseases Section, Children's Specialized Hospital King Saud Medical City, Riyadh, Kingdom of Saudi Arabia. E-mail: o.alzomor@ksmc.med.sa

ORCID ID: https://orcid.org/0000-0002-4016-5163 
$\mathrm{C}^{3}$ arbapenem is a broad-range antibiotic which acts against metallo- $ß$-lactamase (MBL) as well as extended spectrum ß-lactamases (ESBL) produced by many gram-negative pathogens. ${ }^{1-3}$ However, the emergence of carbapenemase-producing carbapenemresistant Enterobacteriaceae (CP-CRE) has threatened the clinical utility of this antibiotic and in fact poses a significant clinical and public health concern. ${ }^{4}$ Klebsiella species and Escherichia coli (E. coli), the common organisms in human gut microbiota, can become carbapenem-resistant. ${ }^{5}$ Klebsiella pneumoniae carbapenemase (KPC) and New Delhi metallo-betalactamase (NDM) are enzymes conferring resistance to carbapenems verona integron-mediated metalloß-lactamase (VIM) from Pseudomonas, imipenemase (IMP) from Enterobacter aerogenes and oxacillinase48-type carbapenemases (OXA-48) from Acinetobacter baumannii are also reported to be carbapenem degrading enzymes. ${ }^{5,6}$ The highest rates of CRE infection are reported in Greece, Italy, Brazil, and China, followed by several other countries including the United States and Colombia. ${ }^{7}$ Oxacillinase-48-type carbapenemases (OXA-48)-producing isolates are commonly observed in cases returning from endemic areas like the Middle East. $^{7}$

Carbapenemase-producing carbapenem-resistant Enterobacteriaceae infections are associated with $\geq 30 \%$ case fatality rates and are typically seen in patients with prior healthcare exposure. ${ }^{8}$ Contaminated medical devices are a common risk factor for CRE acquisition. ${ }^{9}$ Other risk factors are poor functional status, exposure to an intensive care unit and overseas hospitalization. ${ }^{10-12}$ Though sporadic, CRE infections in children are on the rise with an increasing number of cases being reported globally. ${ }^{13}$ Also, little is known about the associated risk factors in this patient population. A 2017 study by Chiotos et al, ${ }^{14}$ showed antipseudomonal antibiotic exposure, prior surgery and mechanical ventilation to be the major risk factors for colonization or infection with CRE in hospitalized children. Thus, rampant use of antibiotics (antibiotic misuse), extensive international travel and poor infection control practices play a role in development of carbapenem-resistance.

In this context, owing to its geographical location, annual hosting of mass gathering events and population flow from the Middle East and India, the Kingdom of Saudi Arabia (KSA) is a potential hotspot for collection

Disclosure. Authors have no conflict of interests, and the work was not supported or funded by any drug company. and spread of these resistance determinants. ${ }^{15} \mathrm{~A}$ recent study by Khan et al, ${ }^{16}$ revealed that Enterobacteriaceae isolates in particular $K$. pneumoniae co-harboring (KPC), NDM-1, and OXA-48 genes are emerging in Western region, KSA. The risk factors for CRE infections identified in this region are: higher mean Charlson comorbidity index (CCI), former antibiotic use, intensive care unit (ICU) admission, and receipt of invasive procedures. ${ }^{17}$ Also, CRE infections registered a higher mortality rate when compared to matched controls $(p=0.031) .{ }^{17}$ Mortality was associated with increased age, presence of comorbidities and length of ICU stay. ${ }^{17}$ However, literature evidence regarding the burden of CRE infections in children from this region is scarce and so is the information on the associated risk factors. Also, effective antibiotic therapy, have not been formally characterized. Thus, this study aims to identify the prevalence and risk factors of infections due to CRE among hospitalized children, aiming to develop effective prevention strategies including strict infection control measures and close adherence to antibiotic stewardship.

Methods. The source population for the study included patients who were hospitalized at King Fahad Medical City (KFMC), Riyadh, KSA between January 2016-2017.

We performed a retrospective matched case-control study to identify the risk factors for CRE colonization or infection in the pediatric population at KFMC. Patients $\leq 17$ years of age, positive for CRE and hospitalized for $\geq 24$ hours were included. Outpatients and patients who had cultures performed in the Emergency department and not admitted were excluded. Subjects were matched for the anatomic site of infection and the causative organism. The study was focused on the first episode of hospital-acquired infection even if recurrent infections occurred. All data were captured retrospectively, and no additional tests were performed. A microbiology laboratory personnel identified the pathogenic CRE (mainly K. pneumonia, E. coli, Enterobacter sp., and Citrobacter sp.) from the microbiology laboratory database. Patients with clinical cultures positive for CRE were included as case-patients. Carbapenemresistant Enterobacteriaceae was defined according to the 2015 Centers for Disease Control and Prevention (CDC) definition. ${ }^{18}$ Patients with positive cultures for carbapenem susceptible Enterobacteriaceae (CSE) were treated as controls. Because of the scarcity of infection with some of the above-stated organisms, controls were selected without randomization. All identified eligible controls were screened for inclusion. Carbapenem resistance and susceptibility were defined per the 
2017 Clinical and Laboratory Standards Institute (CLSI) guidelines. ${ }^{19}$ Two different control groups were selected in order to get a better representation of the total base population. The criteria for selecting control patients were: (i) hospitalization at same institution, (ii) comparable age stratification, (iii) the year of positive culture, (iv) a clinical source of positive culture, and (v) infection versus colonization status. Instances where more than 2 eligible controls were identified for a single case, the ones with the cultures closest in date to that of the case patient were selected. If for a case there were fewer than 2 eligible controls, the case was excluded from the analysis.

Data were collected using a pre-designed data collection sheet adopted from a previous study conducted by Garbati et al. ${ }^{17}$ Collected demographic data included: age, gender, and patient source (home, hospital, long-term care facility). Clinical data included the duration of current hospitalization, site of infection, treatment for the index infection, and the outcome. The severity of illness was assessed by (i) hospitalization within the last 3 months; (ii) patient location at the time of infection; (iii) admission to ICU; (iv) using antibiotics within 3 months before admission; (v) use of central venous catheter (CVC); (vi) use of urinary catheter mechanical ventilation; and (vii) dialysis. Also, the comorbid conditions (cardiovascular disease, lung disease, diabetes mellitus, solid tumors or hematological malignancy, liver disease, renal failure, and chemotherapy) were recorded. The causative organisms isolated from the sites of infection, the date of isolation, and the in vitro susceptibilities of the organisms to various antibiotics, including colistin and tigecycline for participants with more than one episode of infection; data were only collected and analyzed for the first episode. These cases were reviewed to identify the received treatment and also their outcomes. Exposure to several risk factors was considered for the analysis only if it had happened before the acquisition of the infection. Prior antibiotic exposure was considered significant for analysis only if (i) that exposure had occurred within 3 months prior to the hospitalization and (ii) the antibiotic was administered for at least 72 hours.

Articles related to our research were identified through a search of the literature in different databases including Embase, PubMed, GoogleScholar, and ScienceDirect. The search was not limited by country of publication, study design, carbapenem resistance mechanism, or patient characteristics. Studies pertaining to clinical trials, systematic reviews, and meta-analysis, and case reports were included. Articles published only in English language not earlier than 2014 were considered for the drafting of this manuscript.

Ethical approval was obtained from KFMC Institutional Review Board and the research was carried out in accordance to the Declaration of Helsinki.

Statistical analysis. Data were described as mean \pm SD and percentages. Least significant difference will be measured at 95\% CI. Intergroup comparison for metric data were carried out by Student's t-test, whereas Chi-square test and odds ratio will be used for nonmetric variables. Binary logistic regression analysis for multivariate comparison and Kaplan-Meir survival analysis predicted final outcome of the study. Statistical Package for Social Sciences (SPSS) for Windows version 20.0 (IBM Corp., Armonk, NY, USA) was used for data analysis.

Results. Baseline characteristics of the study population. During the one-year study period, 19 cases with index clinical cultures positive for CRE and 38 matched control patients were identified. However, one matched control was excluded because of data incompleteness. The mean age $\pm S D$ of cases was recorded to be $43.9 \pm 46$ months (age range: $2-144$ months) and that for controls was 29.2 \pm 52.2 months (age range: 0-204 months; $p=0.301$ ). There were $56.8 \%$ males among the cases and $57.9 \%$ males among the controls $(p=0.935)$. Forty-seven percent $(\mathrm{n}=9)$ of the cases and $59 \%(n=22)$ of the controls were identified at their home. Cases were admitted into either medical (38.9\%), surgical (11.1\%) or ICU (50\%) wards; among controls the rates of admission in the medical ward was $31.4 \%$, surgical ward was $14.3 \%$ and ICU ward was $54.3 \%$. These values were not statistically different $(p=0.84)$. It was also noted that CRE was associated with increased comorbidities and prolonged hospitalization (Table 1).

Risk factors for colonization or infection with CRE. In a univariate analysis, several variables were associated with colonization or infection with CRE. Compared to matched control patients with CSE, patients with CRE were more likely to have CVC line placement (odds ratio $[\mathrm{OR}]=9.12 ; 95 \%$ confidence interval $[\mathrm{CI}]$ : $[0.97$ 85.77]; $p=0.023$ ), have had a recent surgical procedure $(\mathrm{OR}=3.47 ; 95 \%$ CI: [1.30-9.28]; $p=0.006)$, have undergone any invasive procedures $(\mathrm{OR}=6.48 ; 95 \%$ CI: [1.98-21.18]; $p<0.001)$, use of prior antibiotics $(\mathrm{OR}=5.83 ; 95 \% \mathrm{CI}:[1.38-24.62] ; p=0.008)$ and carbapenem exposure in past 3 months $(\mathrm{OR}=19.73$; 95\% CI: [1.09-12.20]; $p=0.004$; Table 2).

Microbiology of CRE isolates and characterization of the infection sites. Among the CRE index cases, the most commonly identified species were Klebsiella 
Table 1 - Clinical and sociodermographic characteristics of the study participants.

\begin{tabular}{|c|c|c|c|}
\hline Variables & $\begin{array}{c}\text { Case } \\
(\mathrm{n}=19)\end{array}$ & $\begin{array}{c}\text { Control } \\
(\mathrm{n}=37)\end{array}$ & $P$-value \\
\hline $\begin{array}{l}\text { Age }(\text { Mean } \pm S D), \\
{[\text { minimum-maximum] }}\end{array}$ & $\begin{array}{l}43.9 \pm 46 \\
{[2-144]}\end{array}$ & $\begin{array}{c}29.2 \pm 52.2 \\
{[0-204]}\end{array}$ & 0.304 \\
\hline Gender & & & $\neq 0.898$ \\
\hline $\begin{array}{l}\text { Female } \\
\text { Male }\end{array}$ & $\begin{array}{r}8(42.1) \\
11(57.9)\end{array}$ & $\begin{array}{l}16(43.2) \\
21(56.8)\end{array}$ & \\
\hline Admission unit & & & 0.894 \\
\hline $\begin{array}{l}\text { Intensive care } \\
\text { Medical } \\
\text { Surgical }\end{array}$ & $\begin{array}{l}9(50.0) \\
7(38.9) \\
2(11.1)\end{array}$ & $\begin{array}{r}19(54.3) \\
11(31.4) \\
5(14.3)\end{array}$ & \\
\hline Source of patient & & & 0.395 \\
\hline $\begin{array}{l}\text { Home } \\
\text { Hospital } \\
\text { Long-term care facility }\end{array}$ & $\begin{array}{l}9(47.4) \\
7(36.8) \\
3(15.8)\end{array}$ & $\begin{array}{r}22(59.5) \\
13(35.1) \\
2(5.4)\end{array}$ & \\
\hline Source of specimen & & & 0.152 \\
\hline $\begin{array}{l}\text { Body fluid } \\
\text { Urinary tract } \\
\text { Bloodstream } \\
\text { Skin and soft tissue }\end{array}$ & $\begin{array}{l}9(47.4) \\
8(42.1) \\
2(10.5) \\
0 \quad(0)\end{array}$ & $\begin{array}{r}11(29.7) \\
15(40.5) \\
3(8.1) \\
8(21.6)\end{array}$ & \\
\hline Isolated organisms & & & 0.898 \\
\hline $\begin{array}{l}\text { Klebsiella pneumonia } \\
\text { Escherichia coli } \\
\text { Others }\end{array}$ & $\begin{array}{l}9(47.4) \\
6(31.6) \\
4(21.1)\end{array}$ & $\begin{array}{r}18(48.6) \\
13(35.1) \\
6(16.2)\end{array}$ & \\
\hline $\begin{array}{l}\text { Duration of prior } \\
\text { hospitalization in the past } \\
3 \text { months (days) } \pm S D \text { ) } \\
\text { [minimum-maximum] }\end{array}$ & $\begin{array}{c}57.2 \pm 42.9 \\
{[4-90]}\end{array}$ & $\begin{array}{c}28.4 \pm 26.1 \\
{[2-68]}\end{array}$ & 0.003 \\
\hline $\begin{array}{l}\text { Duration of current } \\
\text { hospitalization (mean } \\
\text { days) } \pm \text { SD) [minimum- } \\
\text { maximum] }\end{array}$ & $\begin{array}{c}167 \pm 140.8 \\
{[5-480]}\end{array}$ & $\begin{array}{c}50.4 \pm 73.4 \\
{[1-326]}\end{array}$ & $<0.001$ \\
\hline $\begin{array}{l}\text { Total number of } \\
\text { comorbidities }\end{array}$ & $18(94.7)$ & $33(89.2)$ & 0.491 \\
\hline Number of procedures & $15(78.9)$ & $28(75.7)$ & 0.784 \\
\hline
\end{tabular}

pneumoniae (47.4\%; n=9) and Escherichia coli (31.6\%; $\mathrm{n}=6$ ) while other prevalent species included Enterobacter sp., Citrobacter sp., Kluyvera Ascorbata and Proteus $(21.1 \% ; n=4)$. Similar microbiological profile was seen in matched controls ( $K$. pneumoniae $48.6 \%[n=18]$; E. coli $35.1 \%[\mathrm{n}=13]$ and other species $16.2 \%[\mathrm{n}=6])$.

Infections in body fluids (pleural, pericardial, peritoneal, cerebrospinal, biliary, tracheal, and abscess contents) and wound discharge were the most common (47.4\% each; $n=9)$, followed by urinary tract ( $42.1 \%$; $\mathrm{n}=8)$, and bloodstream infections $(10.5 \% ; \mathrm{n}=2)$. The corresponding outcomes for infection sites among controls were $29.7 \%(\mathrm{n}=11), 40.5 \% \quad(\mathrm{n}=15), 8.1 \%$ $(\mathrm{n}=3)$, and $21.6 \%(\mathrm{n}=8)$.

Mortality was not reported in the CRE group while one patient died in the control group.
Discussion. This study was a single-center retrospective study evaluating risk factors for CRE colonization or infection in children over a period of one-year. Central venous catheter line placement, recent surgery, invasive procedure, prior antibiotic use and carbapenem exposure in past 3 months were significant risk factors for CRE colonization or infection.

In response to increasing multidrug-resistance in the last era in many regions of the Middle East region, this case-control study was conducted at KFMC in Riyadh, $\mathrm{KSA}$, to evaluate our understanding of the prevalent risk factors for CRE colonization or infection among the pediatric age group.

Our study showed that receipt of carbapenem antibiotics in the last 3 months was a significant risk factor for colonization or infection with CRE in children. This is consistent with a United States of America multicenter study with pediatric population which revealed a 3-month exposure to antipseudomonal antibiotics is associated with CRE infection or colonization. ${ }^{14}$ Some available pediatric case series also support this finding. ${ }^{20,21}$ This can likely be attributed to rampant antibiotics use which alters the gastrointestinal flora and results in multidrug resistance. This also highlights the importance of antibiotic stewardship interventions in limiting unnecessary exposure to antibiotics in pediatric cases.

Also, CVC line placement and prior surgery were significantly associated with subsequent CRE colonization or infection, and the association with invasive procedures reached statistical significance. This was in accordance to the study of Chiotos et al. ${ }^{14}$ In one case-control study (from KFMC) about CRE infection among adult hospitalized patients, it was reported that the duration of index admission, prior antibiotic use, ICU stay, and invasive procedures were associated with CRE infection and higher mortality rates. ${ }^{17}$ Most of the literature reported risk factors, are in line with our present study which mainly included prolonged duration of hospitalization, prior surgery, and use of invasive procedures, especially the placement of the CVC line.

Our results revealed that CRE was associated with prolonged hospitalization; however, there was no mortality reported. A 2015 study reported a 30-day mortality rate to be 50\% (23/46) among patients with CRE infections. ${ }^{22}$ Similarly, a study from MiddleWestern part of Taiwan projected a $40.8 \%$ mortality rate. ${ }^{19}$ Study conducted at a single medical center of Northern Taiwan during 2012-2013 showed that a single CRE isolate (with an imipenem minimum inhibitory concentration $[\mathrm{MIC}] \geq 16 \mathrm{mg} / \mathrm{L}$ ) independently 
Table 2 - CRE and CSE infections associated risk factors.

\begin{tabular}{|c|c|c|c|c|}
\hline \multirow[t]{2}{*}{ Characteristics } & CRE $(n=19)$ & $\operatorname{CSE}(n=37)$ & Univariate OR $[95 \% \mathrm{CI}]$ & $P$-value \\
\hline & \multicolumn{3}{|c|}{ n $(\%)$} & \\
\hline \multicolumn{5}{|l|}{ Comorbid conditions } \\
\hline $\begin{array}{l}\text { Pulmonary disease } \\
\text { Cardiovascular disease } \\
\text { Renal disease } \\
\text { Liver disease } \\
\text { Neurologic disease } \\
\text { Malignancy }\end{array}$ & $\begin{array}{l}3(15.8) \\
5(26.3) \\
3(15.8) \\
2(10.5) \\
5(26.3) \\
3(15.8)\end{array}$ & $\begin{array}{c}4(10.8) \\
7(18.9) \\
10(27.0) \\
1(2.7) \\
7(18.9) \\
2(5.4)\end{array}$ & $\begin{array}{c}1.56[0.32-7.48] \\
1.52[0.44-5.28] \\
0.55[0.14-2.19] \\
4.22[0.37-48.63] \\
1.52[0.44-5.28] \\
3.23[0.51-20.52]\end{array}$ & $\begin{array}{l}0.594 \\
0.523 \\
0.346 \\
0.218 \\
0.523 \\
0.197\end{array}$ \\
\hline \multicolumn{5}{|l|}{ Clinical characteristics } \\
\hline $\begin{array}{l}\text { CVC line placement } \\
\text { Urinary catheter } \\
\text { ICU stay } \\
\text { Surgery } \\
\text { Mechanical ventilation } \\
\text { Dialysis } \\
\text { Invasive procedure }\end{array}$ & $\begin{array}{c}4(21.1) \\
3(15.8) \\
9(47.4) \\
13(68.4) \\
5(26.3) \\
0(0) \\
11(57.9)\end{array}$ & $\begin{array}{c}1(2.7) \\
8(21.6) \\
20(54.1) \\
11(29.7) \\
6(16.2) \\
1(2.7) \\
5(13.5)\end{array}$ & $\begin{array}{c}9.12[0.97-85.77] \\
0.72[0.18-2.95] \\
0.86[0.33-2.22] \\
3.47[1.30-9.28] \\
1.81[0.5-6.5] \\
0.95[0.00-54.84] \\
6.48[1.98-21.18]\end{array}$ & $\begin{array}{c}0.023 \\
0.603 \\
0.635 \\
0.006 \\
0.368 \\
0.470 \\
<0.001\end{array}$ \\
\hline \multicolumn{5}{|l|}{ Antibiotic use } \\
\hline $\begin{array}{l}\text { Prior antibiotic use } \\
\text { Penicillin } \\
\text { Aminoglycoside } \\
\text { Carbapenem } \\
\text { Cephalosporin } \\
\text { Quinolone }\end{array}$ & $\begin{array}{l}7(36.8) \\
11(57.9) \\
9(47.4) \\
6(31.6) \\
3(15.8) \\
2(10.5)\end{array}$ & $\begin{array}{c}3(8.1) \\
29(78.4) \\
14(37.8) \\
7(18.9) \\
8(21.6) \\
0(0)\end{array}$ & $\begin{array}{c}5.83[1.38-24.62] \\
0.38[0.11-1.26] \\
1.48[0.48-4.53] \\
1.98[0.56-7.04] \\
0.68[0.16-2.93] \\
8.71[0.37-203.53]\end{array}$ & $\begin{array}{l}0.008 \\
0.108 \\
0.492 \\
0.288 \\
0.603 \\
0.044\end{array}$ \\
\hline Prior Carbapenem exposure in past 3 months & $4(21.1)$ & $0(.0)$ & $19.73[1.09-12.203251]$ & 0.004 \\
\hline
\end{tabular}

predicted 14-day mortality among patients regardless of the isolate was from infection or colonization. ${ }^{23}$ A possible explanation for this is that, though some multidrug-resistant organisms have limited therapeutic options, timely and accurate treatment can benefit the patients.

The results of this study also provide a comprehensive description of the microbiology of pediatric CRE isolates. The most commonly identified isolates included K. pneumonia (47\%), E. coli (31\%), and others (21\%) (Enterobacter sp., Citrobacter sp., Kluyvera Ascorbata and Proteus). This was in accordance to Khan et al, ${ }^{16}$ where it was showed that multidrug-resistant Enterobacteriaceae isolates in particular $K$. pneumoniae co-harboring (KPC), NDM-1 and OXA-48 genes are emerging in Western region, KSA. The findings were also supported by a study from the same region which indicated that in KSA, OXA-48 and NDM-1 are the dominant carbapenemases among the Enterobacteriaceae. ${ }^{24}$

Study limitations. This study has several potential limitations, (i) the retrospective nature of the study which does not guarantee the accuracy and the completeness of the data captured; however, in order to minimize the shortcomings of the retrospective study nature we limited our cohort to inpatients and urged for a comprehensive medical record review by trained physicians; (ii) the sample size in this study is small and warrants more extensive trials; (iii) the results of this study might not be generalizable to other centers as this study was a single-center study; (iv) infected and colonized patients were combined into one group which precluded the evaluation of risk factors (especially for infection). However, since the gastrointestinal tract is generally colonized with antibiotic resistant organisms before the actual infection takes course, the risk factors are likely to be similar. Also, while collecting the data, it was kept in mind that samples isolated from sterile sites and fluids only were to be included and not any isolate without any clinical evidence for infection.

In conclusion, this observational case-control study demonstrates that prior antibiotic exposure (namely carbapenem), recent surgery and use of invasive procedures (in particular CVC) were significant risk factors for colonization or infection with CRE in pediatric population. Also, infection with CRE is also associated with higher morbidity (prolonged hospitalization) as compared with CSE. This underscores the importance of improved hygiene, barrier nursing, continuing education, optimum use of invasive devices, enhanced surveillance, and antimicrobial stewardship in limiting CRE transmission in healthcare facilities. Also, effective antibiotic therapy has not been formerly 
characterized among the pediatric age group where the need for development of novel antimicrobial agents with reliable efficacy against Multidrug-resistant Gramnegative rods is a priority.

Acknowledgment. The authors gratefully acknowledge Ms. Abeer, Microbiology Laboratory; and Mr. Tariq Wani, Research and Center, KFMC, Riyadh, Kingdom of Saudi Arabia for their technical assistance.

\section{References}

1. Codjoe FS, Donkor ES. Carbapenem resistance: a review. Med Sci (Basel) 2017; 6: pii: E1. doi: 10.3390/medsci6010001.

2. Bedenić B, Plečko V, Sardelić S, Uzunović S, Godič Torkar K. Carbapenemases in gram-negative bacteria: laboratory detection and clinical significance. Biomed Res Int 2014; $2014: 841951$.

3. Thapa P, Bhandari D, Shrestha D, Parajuli H, Chaudhary P, Amatya J, et al. A hospital based surveillance of metallobeta-lactamase producing gram negative bacteria in Nepal by imipenem-EDTA disk method. BMC Res Notes 2017; 10: 322.

4. Lutgring JD, Limbago BM. The problem of carbapenemaseproducing-carbapenem-resistant-enterobacteriaceae detection. J Clin Microbiol 2016; 54: 529-534.

5. CDC. Carbapenem-resistant enterobacteriaceae in healthcare settings [Internet]. Centers for Disease Control and Prevention. [Updated 2015; Accessed 2019 July 4]. Available from: https:// www.cdc.gov/hai/organisms/cre/index.html

6. Ding B, Hu F, Yang Y, Guo Q, Huang J, Wang M. Four carbapenem-resistant gram-negative species carrying distinct carbapenemases in a single patient. J Clin Microbiol 2015; 53 : 1031-1033.

7. European Centre for Disease Prevention and Control. Antimicrobial resistance surveillance in Europe 2014 [Internet]. ECDC. [Updated 2015; Accessed 2019 July 4]. Available from: https://ecdc.europa.eu/sites/portal/files/media/en/publications/ Publications/antimicrobial-resistance-europe-2014.pdf

8. Jean SS, Lee NY, Tang HJ, Lu MC, Ko WC, Hsueh PR. Carbapenem-resistant enterobacteriaceae infections: Taiwan aspects. Front Microbiol 2018; 9: 2888.

9. van Loon K, Voor In 't Holt AF, Vos MC. A systematic review and meta-analyses of the clinical epidemiology of carbapenemresistant enterobacteriaceae. Antimicrob Agents Chemother 2017; 62.

10. Centers for Disease Control and Prevention. Carbapenemresistant enterobacteriaceae (CRE) infection: Clinician FAQs. [Updated 2015; Accessed 2019 July 4]. Available from: https:// www.cdc.gov/hai/organisms/cre/cre-clinicianfaq.html

11. Nicolas-Chanoine MH, Vigan M, Laouénan C, Robert J. Risk factors for carbapenem-resistant enterobacteriaceae infections: a French case-control-control study. Eur J Clin Microbiol Infect Dis 2019; 38: 383-393.

12. Ling ML, Tee YM, Tan SG, Amin IM, How KB, Tan KY, et al. Risk factors for acquisition of carbapenem resistant enterobacteriaceae in an acute tertiary care hospital in Singapore. Antimicrob Resist Infect Control 2015; 4: 26.
13. Chiotos K, Han JH, Tamma PD. Carbapenem-resistant enterobacteriaceae infections in children. Curr Infect Dis Rep 2016; 18: 2 .

14. Chiotos K, Tamma PD, Flett KB, Naumann M, Karandikar MV, Bilker WB, et al. Multicenter study of the risk factors for colonization or infection with carbapenem-resistant enterobacteriaceae in children. Antimicrob Agents Chemother 2017; 61: e140.

15. Yezli S, Shibl AM, Memish ZA. The molecular basis of $\beta$-lactamase production in gram-negative bacteria from Saudi Arabia. J Med Microbiol 2015; 64: 127-136.

16. Khan MA, Mohamed AM, Faiz A, Ahmad J. Enterobacterial infection in Saudi Arabia: First record of Klebsiella pneumoniae with triple carbapenemase genes resistance. JIDC 2019; 13: 334-341.

17. Garbati MA, Sakkijha H, Abushaheen A. Infections due to carbapenem resistant enterobacteriaceae among Saudi Arabian hospitalized patients: A matched case-control study. Biomed Res Int 2016; 2016: 3961684.

18. Centers for Disease Control and Prevention. FAQs about choosing and implementing a CRE definition. [Updated 2015; Accessed 2019 July 3]. Available from: https://www.cdc.gov/ hai/organisms/cre/definition.html

19. Huang SR, Liu MF, Lin CF, Shi ZY. Molecular surveillance and clinical outcomes of carbapenem-resistant escherichia coli and klebsiella pneumoniae infections. J Microbiol Immunol Infect 2014; 47: 187-196.

20. Díaz A, Ortiz DC, Trujillo M, Garcés C, Jaimes F, Restrepo AV. Clinical characteristics of carbapenem-resistant klebsiella pneumoniae infections in Ill and colonized children in Colombia. Pediatr Infect Dis J 2016; 35: 237-241.

21. Montagnani C, Prato M, Scolfaro C, Colombo S, Esposito S, Tagliabue C, et al. Carbapenem-resistant enterobacteriaceae infections in children: An Italian retrospective multicenter study. Pediatr Infect Dis J 2016; 35: 862-868.

22. Chang YY, Chuang YC, Siu LK, Wu TL, Lin JC, Lu PL, et al. Clinical features of patients with carbapenem nonsusceptible klebsiella pneumoniae and escherichia coli in intensive care units: a nationwide multicenter study in Taiwan. J Microbiol Immunol Infect 2015; 48: 219-225.

23. Wu PF, Chuang C, Su CF, Lin YT, Chan YJ, Wang FD, et al. High minimum inhibitory concentration of imipenem as a predictor of fatal outcome in patients with carbapenem nonsusceptible Klebsiella pneumoniae. Sci Rep 2016; 6: 32665.

24. Memish ZA, Assiri A, Almasri M, Roshdy H, Hathout H, Kaase M, et al. Molecular characterization of carbapenemase production among gram-negative bacteria in saudi arabia. Microb Drug Resist 2015; 21: 307-314. 